\title{
The Optical System Calibration of the Crop Nitrogen Sensor
}

\author{
Jun Ni, Qianqian Jiang, Zhigang Xu, Weixing Cao and Yan Zhu* \\ National Engineering and Technology Center for Agriculture/Jiangsu key \\ laboratory for Information Agriculture, Nanjing Agriculture University, Nanjing \\ 210095, China \\ nijun@njau.edu.cn,2008101046@njau.edu.cn,xuzhigang@njau.edu.cn, \\ caow@njau.edu.cn,*yanzhu@njau.edu.cn
}

\begin{abstract}
To improve the monitoring accuracy of the crop nitrogen sensor, this paper systematically investigated the optical system parameters affecting the monitoring accuracy of the monitor by analyzing principle of the sensor and the optical system design scheme, and designed a scheme of calibrating optical system parameters which included calibration of single photocell, multi-element combination and integrated optical system. Besides, the parameters calibration of optical system was further conducted by laboratory experiment. The data of rice field experiments were used to validate the effectiveness of the parameter calibration scheme of the optical system. The results indicate that the determination coefficient between normalized difference vegetation index (NDVI) and nitrogen content of rice leaves increases to 0.734 from originally 0.651 . That is, the monitoring performance improves significantly after performing system calibration.
\end{abstract}

Keywords: Crop nitrogen, Sensor, Optical system, Responsivity, Calibration

\section{Introduction}

Crop nitrogen sensor rapidly monitors the status of crop nitrogen nutrition in real-time and provides the implementation of precisely managing crop production with technology support [1]. At present, the monitoring theory of crop nitrogen with non-destructive method, and development of relating devices have been further put forward based on reflectance spectra. American CROPSCAN Company developed the multispectral radiometer for monitoring the reflectance spectra of crop canopy [2]. German Yara Company designed the crop nitrogen content sensor, N-Sensor. A variable rate fertilization group in America prepared the normalized difference vegetation index (NDVI) sensor, Greenseeker [3-4]. American Holland Scientific Company developed a plant canopy spectrometer, Crop Circle ACS-470 [5]. And Japanese TOPCON Company prepared a crop nitrogen content sensor, CropSpec, based on modulated laser [6]. In China, Wang Xiu et al., from Beijing research center for information technology in Agriculture designed a normalized difference vegetation index (NDVI) measuring instrument for managing wheat in field [7-9]. Xia Hui from Anhui institute of optics and fine mechanics developed a dual-wavelength optical sensor to monitor plant nitrogen content based on spectral remote sensing technology [10]. Li Xiuhua et al., from China agricultural university designed a crop canopy analysis instrument based on spectroscopy theory by improving the optical channel and data transmission of the spectroscopy instrument for diagnosing crop growth [11].

The optical system is one of the key components in the crop nitrogen sensor. Its accuracy affects the whole instrument's performance. On one hand, various photoelectric detectors and the differences and attenuations of the performance about each element's

${ }^{*}$ Corresponding Author 
lead to detection errors [12], on the other hand, due to material's particularity, invisible abrasion is likely to happen to optical elements. In addition, monitoring errors possibly occur due to the uncertain factors in assembling process [13]. Presently, standard whiteboard is generally used to calibrate the optical system of the sensor before measurement; however, the abrasion of the standard whiteboard after using for a period of time and the complex calibration operation also affect the measurement accuracy of instrument. However, so far, seldom studies have been made on perfect calibration about optical system of the crop nitrogen sensor.

Aiming at the actual demands for crop nitrogen sensor [14] in field uses, this paper investigated the performance parameters affecting the measurement accuracy of optical system and its corresponding calibration method. Furthermore, the parameter calibration of the optical system was carried out, which provided technological support for improving measurement accuracy.

\section{The Optical System of Crop Nitrogen Sensor}

Crop nitrogen sensor uses sunlight as passive light source. It calculates the canopy spectral reflectance by detecting the incident sunlight and crop canopy reflected light. Then the relation model of spectrum parameter and crop nitrogen is used to calculate the crop nitrogen index. The optical system consists of upward optical sensor and the downward optical sensor, as shown in Figure 1.

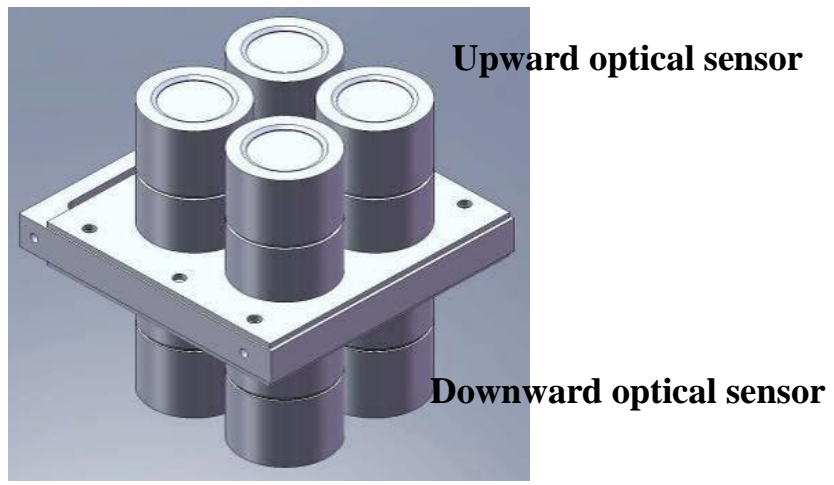

\section{Figure 1. Optical System of the Crop Nitrogen Sensor}

Upward optical sensor is designed to receive solar radiation, including that of the diffuser, band-pass filter, lens and detector. When the sunlight with a radiation flux density $\omega$ radiates on the upward optical sensor at an incidence angle $\theta$, light shoots on the photosensitive surface of the silicon photocell by passing through the diffuser and optical filter, and the output photocurrent $I_{S C U}$ of photocell is [15]

$$
I_{S C U=} S \times H_{\text {upward }}=S \times \beta \times \Omega \operatorname{light}_{1} \times \cos \theta
$$

Where $S$ refers to light responsivity of the photocell in upward optical sensor, A/W;

$H_{\text {upward }}$ refers to illumination value received by the photocell of upward optical sensor, $\mathrm{W} / \mathrm{cm}^{2}$

$B$ refers to attenuation coefficient of upward optical sensor;

$\Omega_{\text {light }}$ denotes the radiation flux density, $\mathrm{W} / \mathrm{cm}^{2}$.

Downward optical sensor is applied to receive canopy reflected light information, including information of window mirror, band-pass filter, lens and detector. When sunlight with radiation flux density $\omega$ irradiates on the crop canopy at an incidence angle $\theta$, crop canopy is an approximate Lambert reflector. The reflected light shoots on the photosensitive surface of the silicon photocell in downward optical sensor by passing 
through window mirror, band-pass filter and photographic lens. The output $I_{S C D}$ of silicon photocell is [15]

$$
I_{S C D}=S^{\prime} \times H_{\text {downward }}=S^{\prime} \times \beta^{\prime} \times N \times \Psi
$$

Where $S$ ' refers to light responsivity of the photocell of downward optical sensor, A/W;

$H_{\text {downward }}$ is illumination value fitting for the photocell of downward optical sensor;

$\beta$ 'refers to attenuation coefficient of downward optical sensor;

$N$ is crop canopy radiance, $\mathrm{W} \cdot \mathrm{sr}^{-1} \cdot \mathrm{cm}^{-2}$;

$\Psi$ denotes field solid angle of downward optical sensor, sr;

Crop canopy approximates to Lambert reflector, on which the hemisphere reflectivity $\rho$ can be calculated by using formula (3) [16-17]

$$
\rho=\pi \times B / E
$$

In the formula, $B$ refers to reflection radiance of diffuse reflectors, $\mathrm{W} \cdot \mathrm{sr}^{-1} \cdot \mathrm{cm}^{-2}$; and $E$ is solar incident irradiance, $\mathrm{W} / \mathrm{cm}^{2}$.

Therefore, $\omega$ in formula (1) and $\mathrm{N}$ in formula (2) are equivalent to $\mathrm{E}$ and $\mathrm{B}$ in formula (3) respectively. By substituting formulae (1) and (2) into formula (3), we obtain

$$
\rho=\pi \times N / \Omega_{\text {light }}=\left[\left(\pi \times I_{S C D}\right) /\left(S^{\prime} \times \beta \times \Psi\right)\right] /\left[I_{S C U} /(S \times \beta \times \cos \theta)\right]
$$

Let $\mathrm{K}=(\pi \times \cos \theta) / \Psi$, and $\mathrm{L}=(S \times \beta) /\left(S^{\prime} \times \beta^{\prime}\right)$, the crop canopy reflectance $\rho$ is

$$
\rho=\mathrm{K} \times \mathrm{L} \times I_{S C D} / I_{S C U}
$$

$\pi$ is a constan, $\psi$ is associated with the field angle and the measuring distance of the optical system; and $\cos \theta$ is related with solar altitude. Those parameters can be obtained by calculation. Absolute values of the parameters in coefficient $L$, which are associated with elements properties of the instrument and optical system structure, cannot be obtain by calculation. They are usually acquired by using standard whiteboard calibration [18]. However, the standard whiteboard tends to be worn after using a period and the complex calibration has to be carried out in each measurement, the measurement accuracy of the sensor is therefore affected. It is necessary to calibrate $L$ in different optical systems of same sensor and different sensors before using instruments. Besides, to ensure that the relations of output and input for optical system do not vary with $L$, optical system of the sensor also needs to be calibrated with $L$.

\section{Optical System Calibration}

\subsection{Calibration Theory of Optical System}

$L$ calibration for optical system includes calibration for the responsivity of each component in optical system and the calibration for attenuation coefficient of optical system for light. Photocell presents different responsivities for incident lights with varied wavelengths, and the responsivity increases with the increase of the wavelength of incident light. Photocell shows larger responsivity and is more sensitive for incident light more than $800 \mathrm{~nm}$ in wavelength. So spectrum responsivity of each element in optical system needs to be adjusted to a same standard before the calibration of elements, so that the responsivity differences arising from the performance differences of each component or attenuation can be eliminated.

The attenuation coefficient of optical system changes with the transmissive wavelength of the band-pass filter. As the band-pass filter has certain bandwidth, the integral responsivity obtained by integral computation for the spectral responsivity within the tranmissive wavelength accurately reflects the attenuation of the optical system for light. The optical system shows a highest responsivity around the core wavelength, where the conversion efficiency for light accounts for more than $80 \%$ of the total wave bands. To 
guarantee the computation accuracy and improve computation efficiency, half-wave width is applied in calculation of the integral responsivity of the optical system. By measuring and calculating the integral responsivity of the optical system, the attenuation coefficient of the overall optical system for light was obtained. In other words, the monitoring errors caused by the structural differences of the optical system and the parameter differences of the components are eliminated, and the monitoring accuracy is therefore improved.

\subsection{Methods for Measuring the Responsivity Parameters of Optical System}

If the irradiances $E(\lambda)$ of the standard detector and measured photocell for an incident light with certain wavelength $\lambda$ are same, their spectral responsivities can be calculated using formulae (6) and (7) respectively.

$$
\begin{aligned}
& R_{\Phi_{s}}(\lambda)=I_{s} / \Phi_{s}=I_{s} / S_{s} \times E(\lambda) \\
& R_{\Phi_{t}}(\lambda)=I_{t} / \Phi_{t}=I_{t} / S_{t} \times E(\lambda)
\end{aligned}
$$

Where $R_{\Phi_{s}}(\lambda)$ and $R_{\Phi_{t}}(\lambda)$ are the spectral responsivities of the standard detector and measured photoelectric components respectively, A/W;

Is and It are the currents generated by the standard detector and measured photocell, A;

$\Phi s$ and $\Phi t$ are the radiation fluxes of the standard detector and measured photocell respectively, W;

Ss and $S t$ are the effective photosensitive areas of the standard detector and the measured photocell, $\mathrm{cm}^{2}$.

As the radiation flux $\Phi t$ of the measured photocell cannot be acquired accurately, the spectral responsivity of the measured photoelectric components is obtained by comparing formula (6) with formula (7), as shown in formula (8)

$$
R_{\Phi_{t}}(\lambda)=\frac{R \Phi_{s}(\lambda) \times I_{t} \times S_{s}}{I_{s} \times S_{t}}
$$

After obtaining the spectral responsivities of the measured photoelectric components in each wave band, the corresponding integral responsivity is obtained by performing integral computation for the spectral responsivities in required wavelength scope.

\section{Calibration Experiments and Results Analysis}

\subsection{Calibration Experiments}

There are four wave bands, 546, 660, 710 and 810nm [19-23] in the optical system of the crop nitrogen sensor. Correspondingly, there are four upward and four downward optical sensors with the filters of the above wave bands. The eight optical sensors were numbered as follow: the upward and downward optical sensors equipped with filters of $546 \mathrm{~nm}$ are marked No.1 and No.2 optical sensors; that with filters of 660nm are marked No.3 and No.4; that with filters of 710nm are marked No.5 and No.6; and that with filters of $810 \mathrm{~nm}$ are marked No.7 and No.8.

According to the principles for measuring the responsivity parameter of optical system, a set of responsivity measuring instrument was designed, as displayed in Figure 2. The components of the eight optical sensors were installed on sample holders, which were placed in the sample room. After chopping by an optical chopper and splitting by a monochromator, the light source entered in the sample room and shot on the components of optical systems. After being scanned by a spectrometer which was controlled by software, the photocurrents generated by the sample and the standard detector were collected using a lock-in amplifier and processed by inputting them in computer; afterward, the absolute value of the spectral responsivity of the sample was acquired by 
comparing with that of the standard detector.

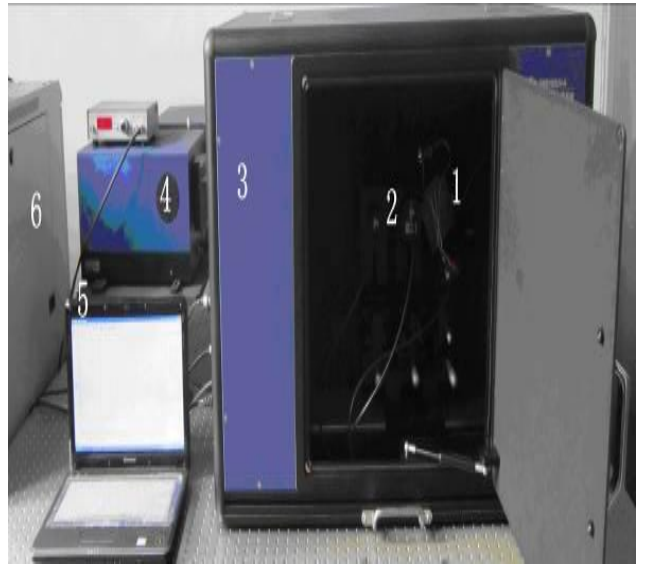

(a) Equipment for Measuring Spectral Response

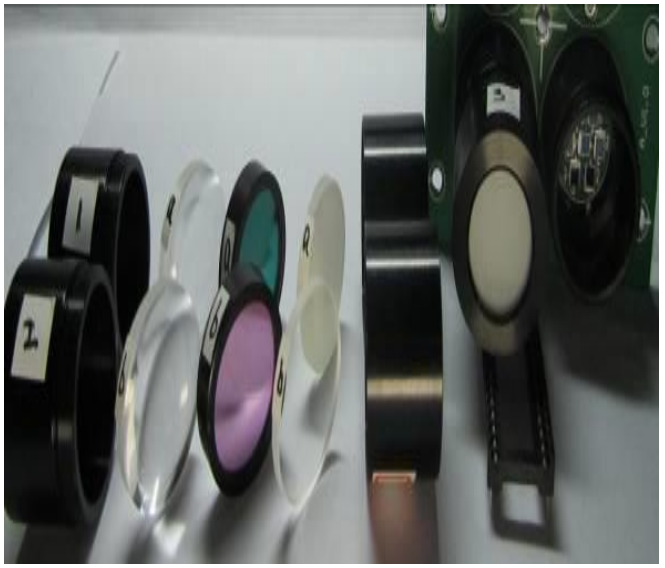

(b) Sensor Components 1. Sample Holder 2. Standard Detector 3. Sample Room 4. Chopper and Monochromator 5. Computer 6. Phase Locked Amplifier

\section{Figure 2. Test Equipment for Calibration}

To cover the spectral scope of the sensor, the accurately measured spectral responsivity parameter in the range of 500-900 $\mathrm{nm}$ was used. To begin with, the spectral responsivity of different silicon photocells were detected. Based on the detection results, the photocells with basically same output of spectral responsivity were discovered and those with different spectral responsivities were changed or their welded surfaces were adjusted. In this way, the responsivity parameters of the photocells used in the instrument were controlled to a same standard. Then the spectral responsivities of different filters, lens and window mirrors (or diffusers) were measured, and the components with inconsistent spectral responsivities were cleaned or replaced to eliminate the measuring errors caused by their abrasion and performance degradation. Finally, the spectral responsivity of the overall optical system was measured and the calibration coefficient was calculated according to the measured result. By calibrating the optical system, the errors caused by the structural difference of the optical sensors and the uncertain factors in assembling process were removed.

\subsection{Results Analysis}

The eight silicon photocells were marked with a, b, c, d, e, f, g and h, and the spectral responsivity curves of them were measured in the range of $500-900 \mathrm{~nm}$, as displayed in Figure 3_a. It demonstrates that the output curve of photocell is abnormally low in the range of 500-800 nm; in the range of 800-900 nm, the output curve of photocell $\mathrm{b}$ is lower than others, the output curves of photocells $\mathrm{e}$ and $\mathrm{h}$ are higher than others, while the deviations about the output curves of other photocells are within $\pm 5 \%$. Therefore, the four silicon photocells, a, b, e and h, with abnormal output curves, were changed and their welded surfaces were adjusted to eliminate significant difference among the spectral responsivity curves of all the silicon photocells, as illustrated in Figure 3b. Regarding the remaining output differences, they were calibrated in the final computation of calibration. 

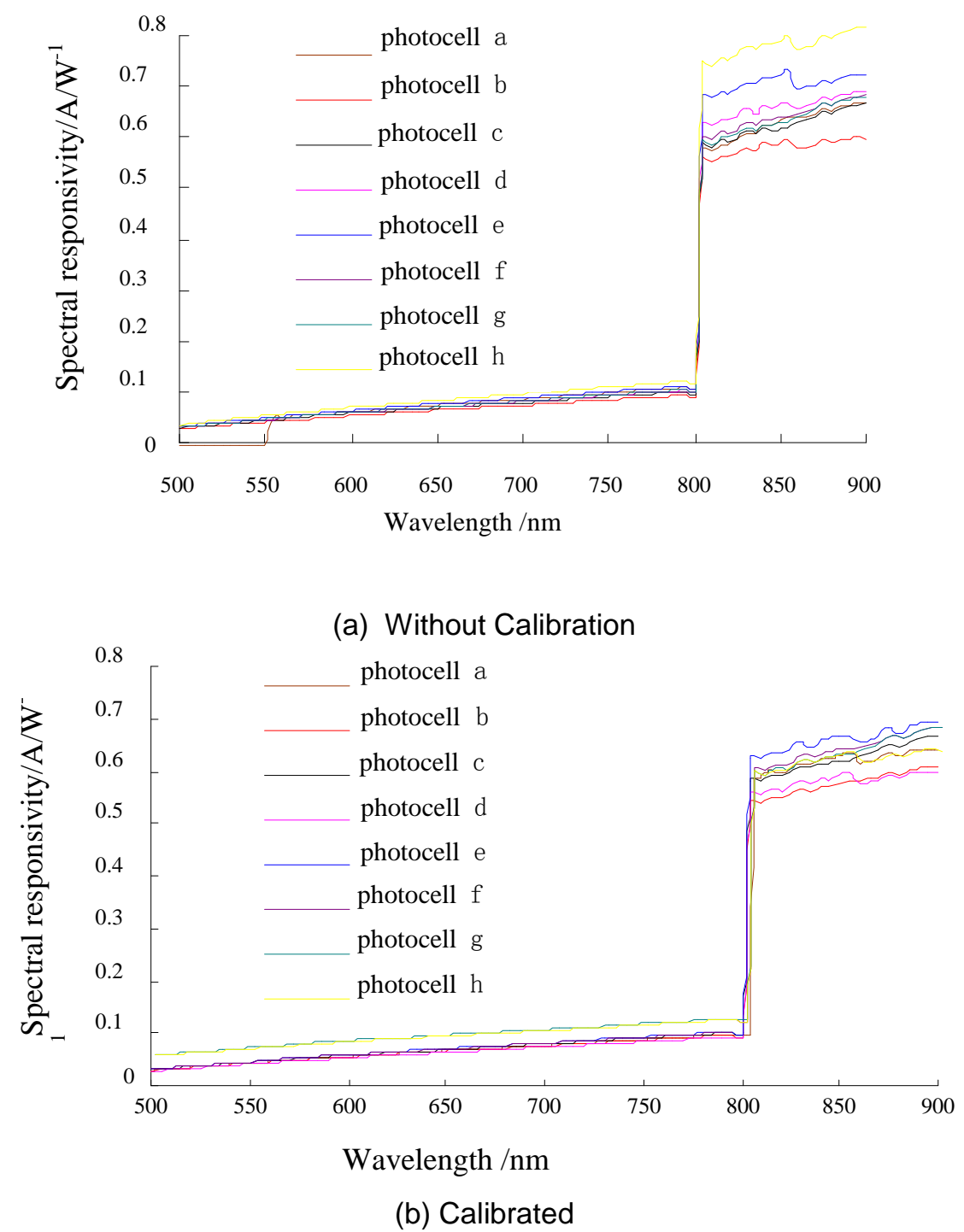

Figure 3. Spectral Responsivity of Photocell

A set of photocell panel with adjusted welded surfaces was used randomly. Eight lens marked with a, b, c, d, e, f, g and h were cleaned. The filters that $546 \mathrm{~nm}$ in wavelength were assembled on lens $a$ and $b$, the filters of $660 \mathrm{~nm}$ were assembled on lens $c$ and d, the filters of $710 \mathrm{~nm}$ were assembled on lens e and $\mathrm{f}$, and the filters of $810 \mathrm{~nm}$ were assembled on lens $g$ and $h$. The spectral responsivity curves of the photocells with different band-pass filters and lens were measured, as shown in Figure 4. 


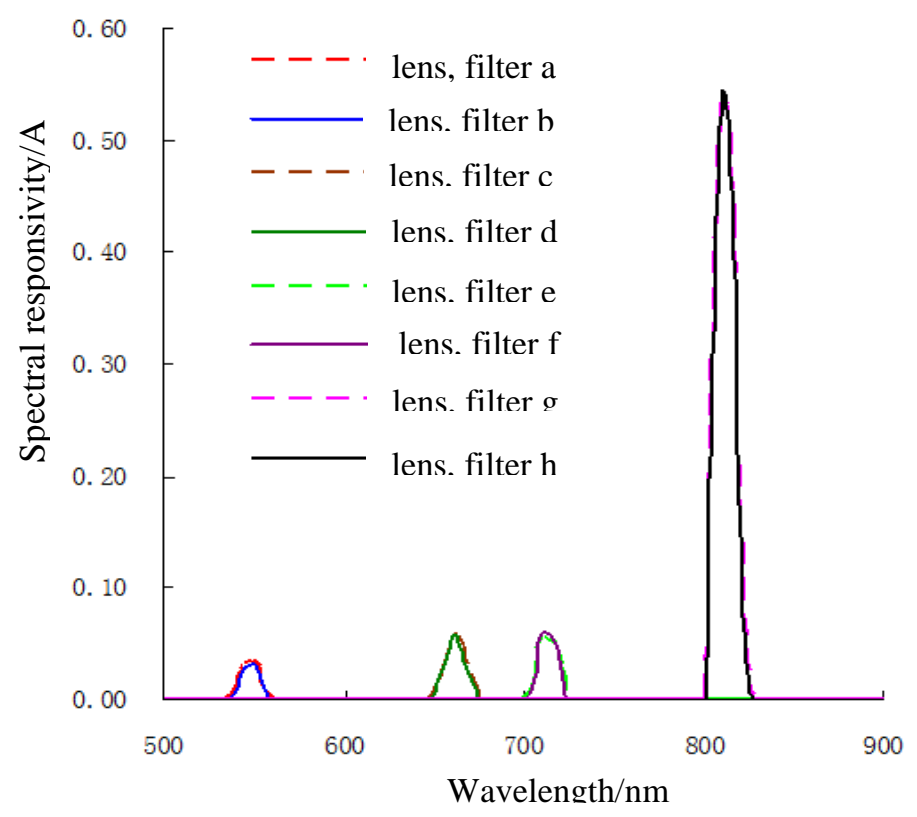

\section{Figure 4. Spectral Responsivity of Calibrated Photocell Assembling Different Filters and Lens}

Figure 4 indicates that the peak wave of the spectral responsivity of different filters was majorly discovered at the characteristic wavelengths of the filters, and the other wave bands show different attenuations for light. In contrast, the attenuations of filters with same wavelength are basically same. If the lens or filters were stained or improperly assembled, their measured spectral responsivities were different. So, the lens or filters with inconsistent spectral responsivities have to be cleaned, changed or adjusted to guarantee the conformity of the spectral responsivities of different lens and filters in their corresponding characteristic bands.

Four diffusers and four window mirrors were numbered as a, b, c and d, and installed on the silicon photocells used in the above experiments, respectively. The spectral responsivity curves of silicon photocells with different window mirrors (or diffusers) were measured, as stated in Figure 5.

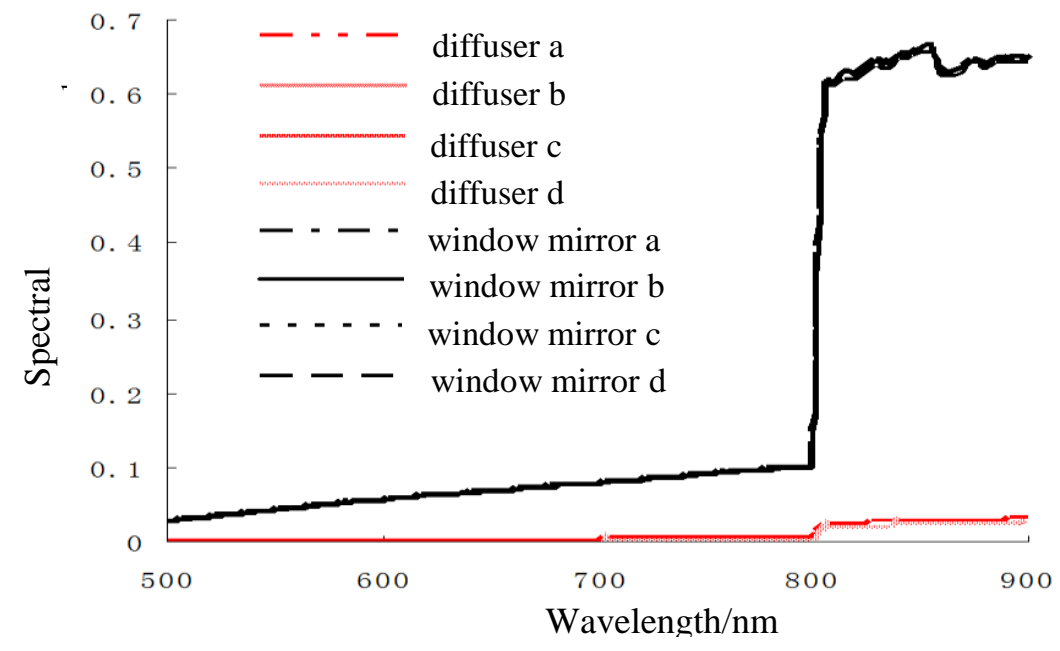

Figure 5. Spectral Responsiveness of Calibrated Photocell Assembling 


\section{Different Window Mirrors (Or Diffusers)}

Figure 5 illustrates that all the spectral responsivity curves of the diffusers (or window mirrors) were basically consistent; the diffusers present distinct attenuation effect; and the window mirrors exhibit small attenuation for light. Deviation of spectral responsivities was measured if the window mirrors or diffusers were stained or improperly assembled. In the situation, the window mirrors or diffusers with inconsistent spectral responsivities have to be cleaned, changed or adjusted to eliminate the errors resulted from the performance attenuation of different window mirrors or diffusers or the errors in assembling process.

Following the order of the number, the photocells, lens, filters and window mirrors (or diffusers) were assembled, and the overall spectral responsivity of the eight optical sensors were measured, as demonstrated in Figure 6.

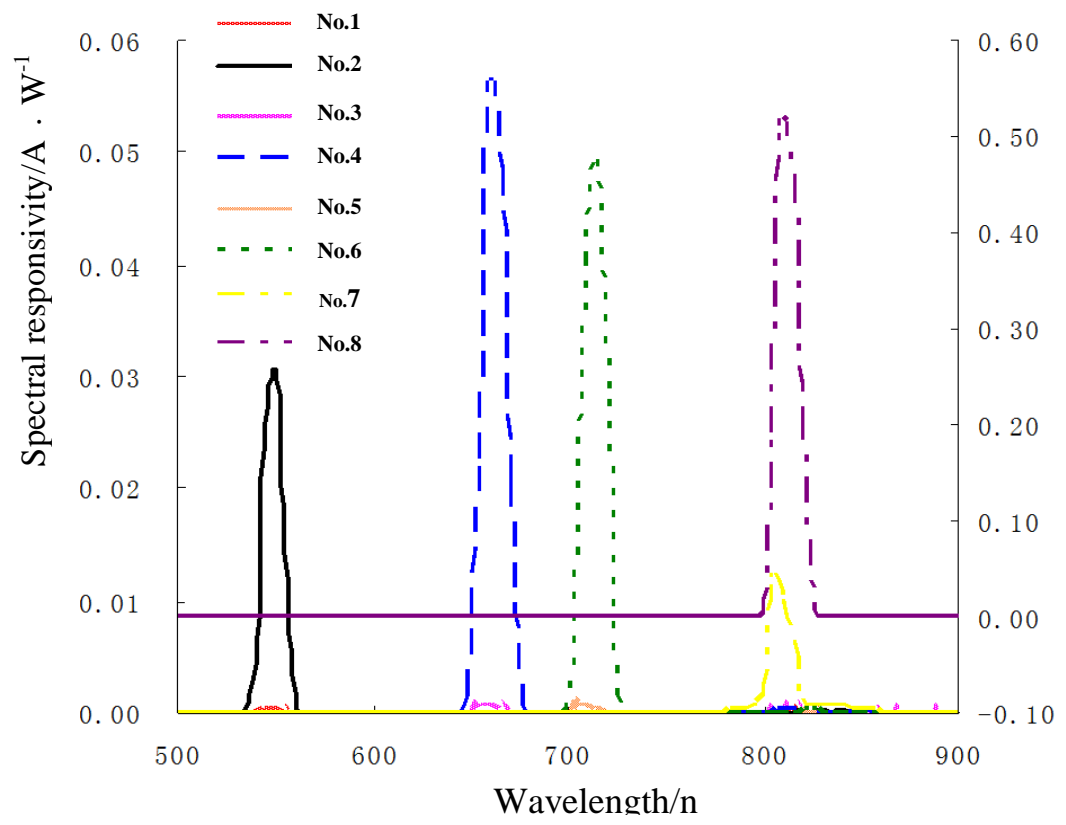

Figure 6. Spectral Responsitivity of the Optical System

In Figure 6, the peak waves of all the spectral responsivities of the optical sensors containing filters in different wave lengths were observed at the core wavelengths; and the attenuations of the upward and downward optical sensors that contain filters in same wave length are different owing to the different performances of window mirrors and diffusers.

Based on the spectral responsivities measured in Figure 6, the peak waves and halfwave widths of each optical sensor were calculated, as well as the integral responsivity of half-wave width, as displayed in Tables 1 and 2.

Table 1. Parameters about Spectral Response of Upward Optical Sensor

\begin{tabular}{ccccc}
\hline & \multicolumn{4}{c}{ Wavelength $(\mathrm{nm})$} \\
\cline { 2 - 5 } Upward optical sensor & 546 & 660 & 710 & 810 \\
\hline Peak value/A $\cdot \mathrm{W}^{-1}$ & 0.001 & 0.001 & 0.001 & 0.012 \\
Center wavelength & 545 & 660 & 710 & 805 \\
/nm & 539.532 & 651.344 & 702.022 & 802.149 \\
Half wavelength /nm & 552.490 & 666.111 & 717.420 & 815.122
\end{tabular}


Integral response /A $\mathrm{W}^{-1}$

Table 2. Parameters about Spectral Response of Downward Optical Sensor

\begin{tabular}{ccccc}
\hline \multirow{2}{*}{$\begin{array}{c}\text { Downward optical } \\
\text { sensor }\end{array}$} & 546 & 660 & 710 & 810 \\
\cline { 2 - 5 } & 0.031 & 0.056 & 0.049 & 0.512 \\
\hline Peak value/A $\bullet \mathrm{W}^{-1}$ & 550 & 660 & 715 & 810 \\
Center wavelength /nm & 542.56 & & & \\
& 4 & 655.752 & 707.555 & 805.991 \\
Half wavelength /nm & 555.23 & 668.732 & 720.653 & 819.091 \\
& 2 & & & \\
Integral response /A & 0.313 & 0.579 & 0.614 & 5.417 \\
W $^{-1}$ & & &
\end{tabular}

Table 1 and Table 2 indicate that the integral responsivity ratio of the upward optical sensors of $546 \mathrm{~nm}, 660 \mathrm{~nm}, 710 \mathrm{~nm}$ and $810 \mathrm{~nm}$ to the downward ones are 1/44.71, $1 / 44.53,1 / 43.85$ and $1 / 43.68$ respectively. The values, that are, the optical calibration coefficients, reflect the combined influences of the responsivity and attenuation of the upward and downward optical sensors in corresponding wave bands. By calibrating $L$ values of each optical sensor using these calibration coefficients and inputting them in the processing system, the measurement errors caused by parameter difference of optical sensor were eliminated and the measurement accuracy of the sensor was therefore improved.

\subsection{Field Experiments}

To verify the calibration effect of the optical sensor, experiments were carried out for monitoring the nitrogen of rice canopy at the experimental station $\left(32^{\circ} 16^{\prime} \mathrm{N}, 119^{\circ} 11^{\prime} \mathrm{E}\right)$ of Xinji town, Yizheng city, Jiangsu province, China during June to December of 2010. Two crop nitrogen sensors with uncalibrated and calibrated optical systems were used in the experiments to measure the reflection spectra of the rice with different nitrogen contents. The experiments were carried out in sunny days during 10:00 to 14:00 (with a solar altitude larger than $35^{\circ}$ ). Standard white board calibration was performed for the uncalibrated sensor before collecting objective spectra, and for the calibrated monitor, the longitude and latitude of the measuring position were input to calculate the incident angle of sunlight. In the measurement, the optical systems of the sensors were about $1 \mathrm{~m}$ in height vertically to the rice canopy and six times of measurements were performed for each area. The average value of the measurements was used as the measured value of the spectra in the area and the NDVI $(810,660)$ at $660 \mathrm{~nm}$ and $810 \mathrm{~nm}$ was calculated. Simultaneous to the measurement for the spectra of rice canopy, micro-Kjeldahl method was applied to test the nitrogen content of canopy leaves. Afterward, the relation between NDVI $(810,660)$ and the nitrogen content of rice canopy leaves was analyzed and the results were demonstrated in Figures 7 and 8. 


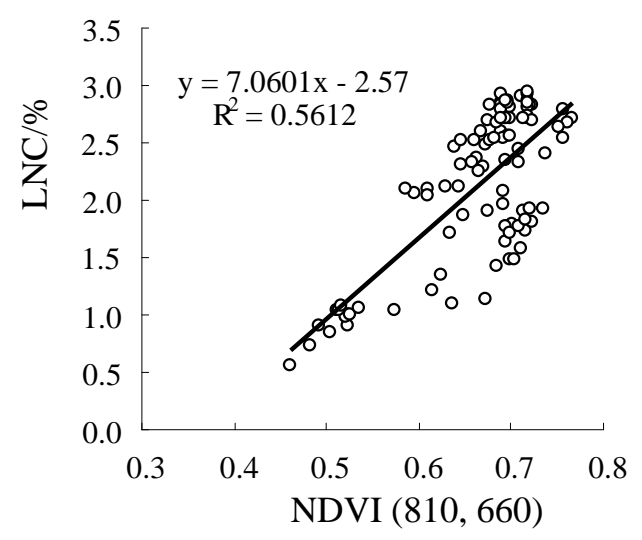

Figure 7. Relationship between Leaf Nitrogen Content of Rice and NDVI (810, 610) Without Calibration

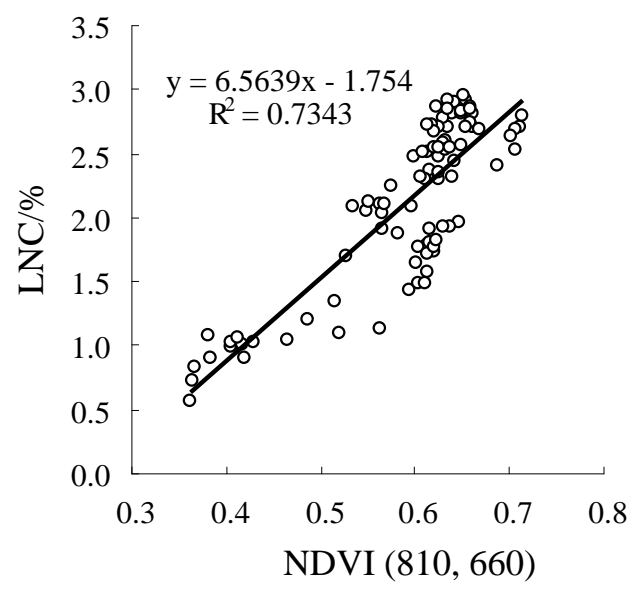

Figure 8. Relationship between Leaf Nitrogen Content of Rice and Calibrated NDVI $(810,610)$

The comparison of Figure 7 and Figure 8 illustrates that the determination coefficient of the NDVI $(810,660)$ and the nitrogen content of rice leaves measured using the calibrated sensor increases from 0.5612 to 0.7374 , and the variance decreases from 0.056 to 0.037. It reveals that the calibration of optical system improved the monitoring accuracy of the sensor.

\section{Conclusions}

The spectral responsivity of each component and each component combination (overall optical system) in the optical system were measured, and the components and assembling position were adjusted to eliminate the errors caused by the abrasion and performance attenuation of components; then by calculating the integral responsivity of the overall optical system, the measuring errors resulted from the structural difference of upward and downward optical sensors and uncertain factors in assembling were eliminated; finally, the calibrated optical system was tested by carrying out field experiments. The results indicated that the determination coefficient of the NDVI $(810,660)$ and the nitrogen content of rice leaves measured using the calibrated sensor increases from 0.56 to 0.74 , and the variance decreases from 0.056 to 0.037 . The significant improvement of the monitoring performance provided technological support for further improving the monitoring accuracy of crop nitrogen. 


\section{Acknowledgements}

The authors would like to express their gratitude to all those who helped in this research. This project was also supported by the National Natural Science Foundation of China (Grant No. 31371534), Jiangsu Agriculture Science and Technology Innovation Fund, (Grant No. CX (12)3049) and Priority Academic Program Development of Jiangsu Higher Education Institutions (PAPD).

\section{Reference}

[1] X. Zhigang, Z. Yan and J. Xuelei, "Design of Optic System for Crop Nitrogen Non-destructive Monitoring Instrument", Transactions of the Chinese Society for Agricultural Machinery, vol. 39, no. 3, (2008), pp. 120-124.

[2] Z. Yan, L. Ying-Xue and Z. Dong-Qin, "Quantitative Relationship between Leaf Nitrogen Concentration and Canopy Reflectance Spectra in Rice and Wheat", Acta Ecologica Sinica, vol. 26, no. 10, (2006), pp: 3463-3469.

[3] A. M. Ali, H. S. Thind, S. Sharma and Varinderpal-Singh, "Prediction of Dry Direct-Seeded Rice Yields Using Chlorophyll Meter, Leaf Colour Chart and Greenseeker Optical Sensor in Northwestern India”, Field Crops Research, vol. 161, no. 5, (2014), pp. 11-15.

[4] O. S. Alsh, A. R. Klatt, J. B. Solie, C. B. Godsey and W. R. Raun, "Use of Soil Moisture Data for Refined Greenseeker Sensor Based Nitrogen Recommendations in Winter Wheat", Precision Agriculture, vol. 14, no. 3, (2013), pp: 343-356.

[5] D. W. Lamb, M. G. Trotter and D. A. Schneider, "Ultra Low-level Airborne (ULLA) Sensing of Crop canopy Reflectance: A Case Study Using a CropCircle ${ }^{\mathrm{TM}}$ Sensor", Computers and Electronics in Agriculture. vol. 69, no. 1, (2009), pp: 86-91.

[6] S. Reusch, J. Jasper and A. Link, "Estimating Crop Biomass and Nitrogen Uptake Using Cropspectm, A Newly Developed Active Crop-Canopy Reflectance Sensor", International Conference on Precision Agriculture, Denver, Colorado USA, (2010) July 18-21.

[7] W. Xiu, Z. Chunjiang and Z. Hanchang, "Development and Experiment of Portable NDVI Instrument for Estimating Growth Condition of Winter Wheat", Transaction of the CSAE, vol. 20, no. 4, (2004), pp. 95-98.

[8] H. Xiaojian, H. Lina and L. Liangyun, "A Intelligent Portable Instrument for Measuring NDVI", Chinese Journal of Scientific Instrument, vol. 26, no. 3, (2005), pp: 246-248.

[9] Z. Chunjiang, L. Liangyun and Z. Hanliang, "Development and Application of A Novel NDVI Instrument", Optcal Technique, vol. 30, no. 3, (2004), pp. 324-329.

[10] W. Xiaomei, L. Hongbin and L. Jianguo, "The Development of Multiple Wavelength Optical Remote Sensing System for Monitoring Plant Nitrogen Concentration", Journal of, Atmospheric and Environmental Optics, vol. 7, no. 7, (2007), pp. 64-68.

[11] L. Xiuhua and L. Minzan, "Non-destructive Crop Canopy Analyzer Based on Spectral Principle", Transactions of the Chinese Society for Agricultural Machinery, vol. 40, suppl, (2009), pp. 252-255.

[12] F. Sakuma and A. Ono, "Radiometric Calibration of the EOS ASTER Instrument", Metrologia, vol. 30, no. 4, (1993), pp. 231-241.

[13] A. Ono and F. Sakuma, "ASTER Calibration Concept", Future European and Japanese Remote-Sensing Sensors and Programs, Orlando, Florida USA, (1991) April 1-2.

[14] J. Xuelei, C. Weixing and T. Yongchao, "Feeble Light Signal Conversion Method and Circuit Design Used in Crop Spectrum Information Inspection", Chinese Journal of Scientific Instrument, vol. 29, no. 11, (2008), pp. 2461-2464.

[15] P. U. Zhaobang, "Photoelectric Testing Technology", Beijing: Machinery Industry Press, (2005), pp. 6367.

[16] J. Zhonghui, "The Application of the Reflect Spectrum Testing Principle on Agriculture", Physics, vol. 21, no. 4, (1992), pp. 225-231.

[17] K. Zhou, "Applied Optics", Chengdou: Sichuan University Press, (2007).

[18] T. Xiujin and Z. Xiaodong, "The Application of Normal White Board on Color Metrology", The Metrology Technology, vol. 6, no. 4, (1996), pp. 35-37.

[19] K. F. Bronson, T. T. Chua and J. D. Booker, "In-Season Nitrogen Status Sensing in Irrigated Cotton: II. Leaf Nitrogen and Biomass", Soil Science Society of America Journal, vol. 67, no. 5, (2003), pp. 14391448.

[20] B. J. Yoder and R. E. Pettigrew-Crosby, "Predicting Nitrogen and Chlorophyll Concentrations from Reflectance Spectra (400-2500nm) at Leaf and Canopy Scales", Remote Sensing of Environment, vol. 53, no. 3, (1995), pp. 199-211.

[21] X. Lh, C. Wx and L. Wh, "Monitoring Leaf Nitrogen Status in Rice With Canopy Spectral Reflectance" Agronomy Journal, vol. 96, no. 1, (2004), pp. 135-142. 
[22] R. F. Kokaly and R. N. Clark, "Spectroscopic Determination of Leaf Biochemistry Using Band-Depth Analysis of Absorption Features and Stepwise Multiple Linear Regression", Remote Sensing of Environment. vol. 67, no. 3, (1999), pp. 267-287.

[23] R. F. Kokaly, "Investigating a Physical Basis for Spectroscopic Estimates of Leaf Nitrogen Concentration”, Remote Sensing of Environment, vol. 75, no. 2, (2001), pp. 153-161.

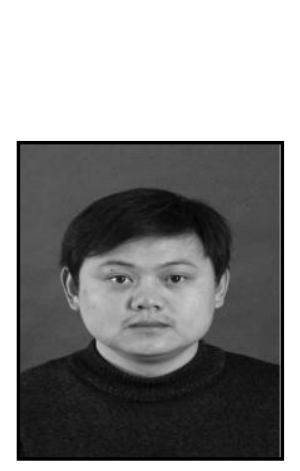

\begin{abstract}
Authors
Jun Ni, he is currently an Associate Professor in the National Engineering and Technology Center for Agriculture at the Nanjing Agricultural University, China. In 2009, he received the $\mathrm{Ph} . \mathrm{D}$. in agriculture engineering field. His research interests include agricultural sensor, agricultural automation system.
\end{abstract}

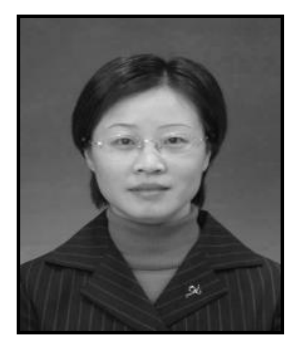

Yan Zhu, she received her $\mathrm{PhD}$ degree from the Nanjing Agricultural University in 2003. Currently, she is a full professor and the dean of College of Agriculture at NJAU. Prof. Zhu works mainly on information agriculture, specifically on crop modeling and crop monitoring. 